\title{
SENSITIVITY OF DETECTION RESPONSE TASK (DRT) TO THE DRIVING DEMAND AND TASK DIFFICULTY
}

\author{
Marie-Pierre Bruyas \& Laëtitia Dumont \\ IFSTTAR-LESCOT \\ Bron, France \\ Email: marie-pierre.bruyas@ifsttar.fr
}

\begin{abstract}
Summary: The Detection Response Task (DRT) is currently discussed in the ISO working group TC22/SC13/WG8 as the basis of a standard to assess the effect of cognitive load on driver attention. This paper investigates the sensitivity of the method to cognitive and visual-manual tasks of different levels of difficulty and to different levels of driving demand. Three versions of DRT have been used in a simulator experiment: two visual versions (HDRT and RDRT) and one tactile version (TDRT). The results show that response times to DRT stimuli increase with the driving demand and with the difficulty of the cognitive auditory task. However, no difference is registered between visual-manual tasks of different levels of difficulty, which is explained in terms of attentional allocation and ceiling effect.
\end{abstract}

\section{INTRODUCTION}

In recent years, the development of tools aiming at measuring the effect that in-vehicle information systems may have on road safety has received much attention, especially in the context of driver's distraction. Different methods have already been standardized, such as the Occlusion method or the Lane Change Test (LCT). The Occlusion method, originally designed by Senders et al. (1967) proposes a procedure for measuring visual demand due to the use of visual displays. The procedure consists in intermittent viewing of in-vehicle systems and provides an estimation of the task interruptibility (ISO TS 16673, 2006). The LCT is intended to estimate secondary task demand on the driver, by measuring performance degradation on a primary driving-like task. The driving performance degradation is inferred from the deviation from an ideal pathway measurement calculated during the lane changes (ISO 26022, 2010; Mattes and Hallen, 2009). Both tools have the advantages to be inexpensive, quick to complete, easy to administer and capable of measuring aspects of drivers' distraction. In this framework, the Detection Response Task is currently being discussed in the ISO working group TC22/SC13/WG8 as the basis of a standard to assess the effect of cognitive load on driver attention. This tool derives from the Peripheral Detection Task, which was intended to measure the cognitive attentional resources that are available to drivers when performing secondary tasks (Jahn et al., 2005). The DRT method consists in a simple detection-response task where participants respond to frequent stimuli presented according to different modalities.

The objective of the present study is to investigate the sensitivity of the DRT to the driving demand and to the level of difficulty of secondary tasks. For this purpose, two driving scenarios of different demand have been designed and secondary tasks of two modalities (cognitive auditory and visual-manual) have been used. Finally, three versions of the DRT were considered: 
two visual versions (HDRT and RDRT) and one tactile version (TDRT), which are described below.

\section{METHOD}

\section{Participants}

Sixteen participants have been recruited (eight males and eight females). All participants were aged between 25 and 45 years (mean age 35.2; SD 5.4). All of them had a driving license and reported driving at least $10000 \mathrm{~km}$ per year. A compensation of $60 €$ was paid for their participation in the experiment.

\section{Driving scenarios}

The experiment was carried out in the IFSTTAR fixed-based simulator. The road scene was displayed on five screens $\left(220^{*} 165 \mathrm{~cm}\right)$ which delivered together $180^{\circ}$ horizontal visual field and $40^{\circ}$ vertical one. The car body was a Peugeot 308 with manual gearbox and all standard passenger compartment features, displays and controls.

Two driving scenarios have been used. The first one consisted in a free driving on a $2 * 2$ lane motorway, with some ambient traffic not interacting with the subject vehicle. Here, the drivers were instructed to respect the speed limit of $130 \mathrm{~km} / \mathrm{h}$. In the second scenario, series of curves of 60 seconds' duration were separated by straight road sections of at least 30 seconds each. A lead car preceded the subject vehicle such that the drivers did not exceed $75 \mathrm{~km} / \mathrm{h}$ in the curves. The drivers received the instruction to give priority to the primary task of driving and to do their best to perform DRT and secondary tasks simultaneously.

\section{DRT versions}

Three DRT versions have been used: Head-mounted DRT, Tactile DRT and Remote DRT. The two first ones were displayed by using a device supplied by TNO. In the Head-mounted DRT (HDRT), visual stimuli were presented by means of a single red LED attached to the head as shown in Figure 1. In the Tactile DRT (TDRT), tactile stimuli were presented by means of a small electrical vibrator. The tactor was placed on the driver's left clavicle as shown in Figure 2.

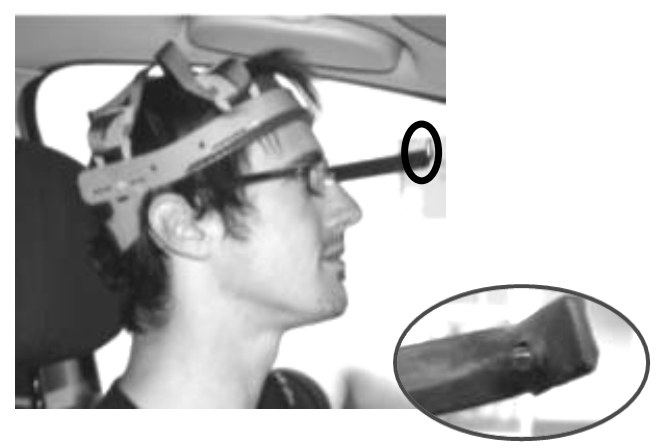

Figure 1. TNO Head-mounted device

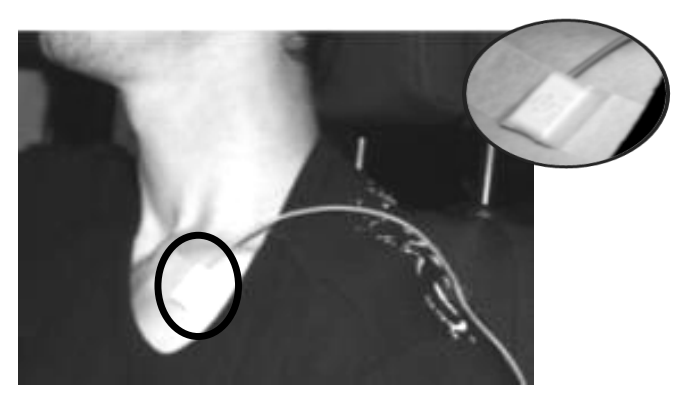

Figure 2. TNO Tactile device 
In the remote DRT, a red LED of $5 \mathrm{~mm}$ was fixed remotely from the participant on the windscreen behind the steering wheel (visual angle $10^{\circ}$ ), so that it could be perceived easily by the driver being close to his/her central field of view (Figure 3).

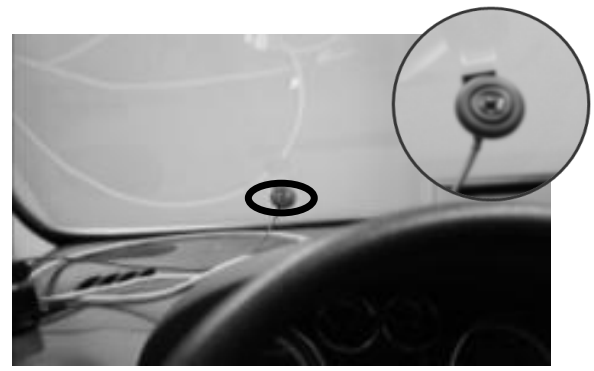

Figure 3: Remote device

\section{Secondary tasks}

Two types of secondary tasks were performed at two levels of difficulty.

Cognitive auditory tasks. The N-Back task has been used (Mehler, Reimer \& Dusek, 2011). This task consisted of recorded series of 10 digits that the drivers had to listen to. In the easy condition (N0), the drivers simply repeated aloud the last number heard. In the harder condition (N1), they had to repeat the number before the last number heard. In both conditions, the participants have been instructed to be as accurate as they can be.

Visual-manual tasks. Two levels of the Surrogate Reference Task (SuRT) have been used (ISO TS 26022). In order to perform these tasks, a screen was fixed on the dashboard, and a numeric keypad was installed below, so that the drivers could carry out the commands related to the tasks. The objective of the task was to look at the screen and to locate a target (big circle) among distractors (smaller circles). To select the target, the participants moved a cursor to the relevant zone by using right and left arrows, then they validated the choice and a new configuration was given by the system. Three levels of difficulty could be activated. Level easy (S0) and level hard (S1) were used in this experiment. The two levels differed in terms of target size and number of zones included in the screen (Figures $4 \mathrm{a} \& 4 \mathrm{~b}$ ).
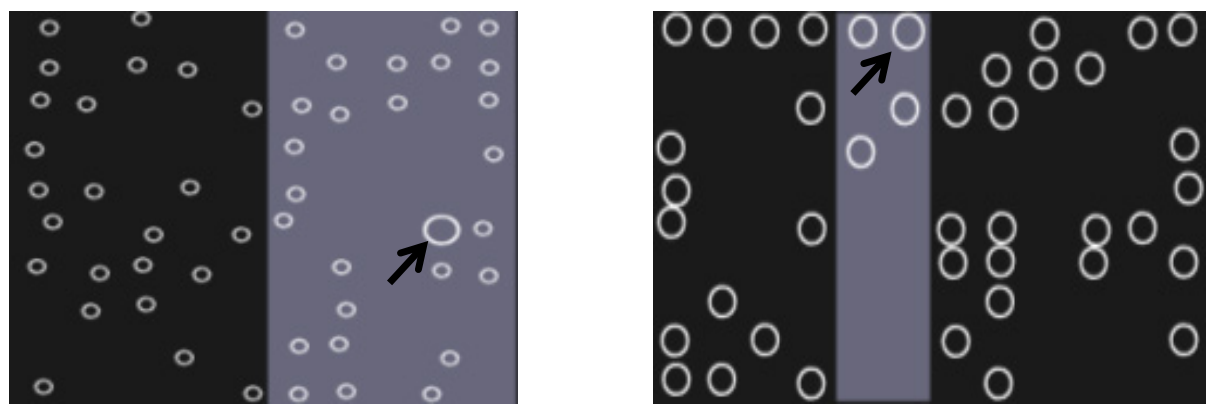

Figures 4a: SuRT easy (S0) \& 4b: SuRT hard (S1) 


\section{Experimental design}

A repeated measures experimental design has been used. All participants performed the five task conditions (baseline, N-Back easy and hard, SuRT easy and hard) while using successively the three versions of DRT (HDRT, TDRT, RDRT) and without any DRT; first driving in the highway scenario and then driving in the curvy road scenario.

Each drive (series of 5 tasks using one DRT version or without DRT) was performed continuously during 12 to 15 minutes, depending on the scenario and the participants' speed. The task duration was of 60 seconds for each task with an interval of at least 30 seconds between tasks. After each drive, the participants filled a questionnaire which allowed for collecting information about their experience during the experiment (difficulties, simulator sickness...). The order of DRT conditions was counterbalanced across the two driving scenarios and the order of tasks was counterbalanced across the four DRT conditions.

\section{RESULTS}

\section{DRT response times (Figure 5)}

Performances for DRT have been calculated in terms of response times (RT) for the three DRT versions. A significant effect of the driving task demand was shown $[\mathrm{F}(1,15)=16.809$, $p=0.001)]$. RTs were significantly higher in the curvy road scenario than in the highway. A significant effect of DRT versions was also shown $[\mathrm{F}(2,14)=24.414, p<0.001)]$. RTs for TDRT were significantly lower than for the 2 other versions $(p<0.05)$. No significant difference was found between HDRT and RDRT versions.

RTs varied significantly according to the tasks $[F(4,12)=29.579, p<0.001)]$. All tasks differed from one another except SURT easy (S0) and hard (S1). Tasks could then be sorted as follow from the significantly lowest RTs to the significantly highest RTs: Baseline, N-Back0, N-Back1 and both SuRT (easy and hard) $(\mathrm{p}<0.05)$.

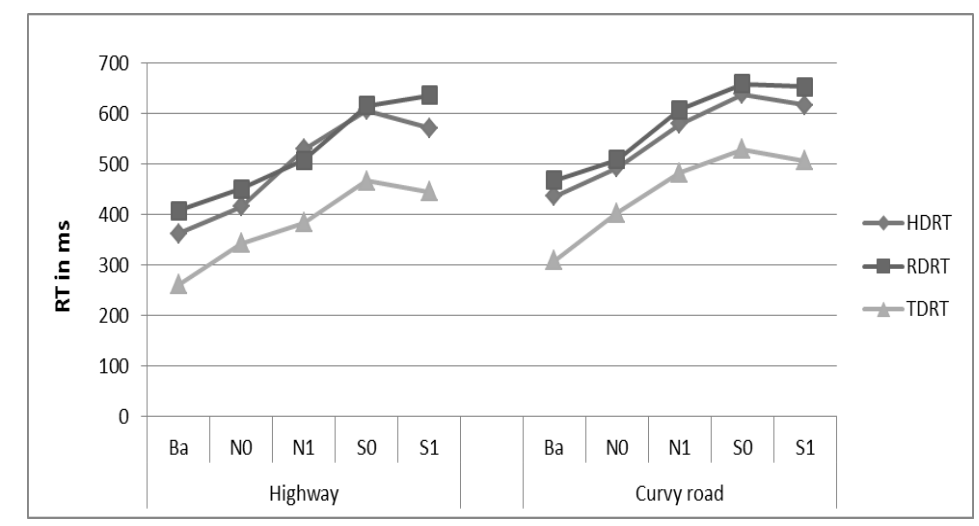

Figure 5. DRT RT for each task and driving scenario 


\section{SuRT response times (Figure 6)}

Performances for SuRT were calculated in terms of response times (RT) defined as the time from stimulus onset to the keypress of the confirmation key. RTs were calculated for the three DRT versions and also for one driving condition without DRT.

A significant effect of the driving task demand was shown $[\mathrm{F}(1,15)=17.386, p=0.001)]$. RTs were significantly higher in the curvy road scenario than in the highway scenario.

A significant effect of DRT versions was also shown $[\mathrm{F}(3,13)=3.683, p=0.041)]$ but pairwise comparisons did not show any difference between the DRT conditions.

RTs varied significantly according to the task levels of difficulty $[\mathrm{F}(1,15)=74.633, p<0.001)]$. RTs for SuRT easy were significantly lower than for SuRT hard $(p<0.05)$.

Moreover, a significant interaction between task levels of complexity and driving scenarios was highlighted showing that the increase of RT from SuRT easy to SuRT hard was even greater in the curvy road scenario than in the highway $[\mathrm{F}(1,15)=17.244, p=0.001)]$.

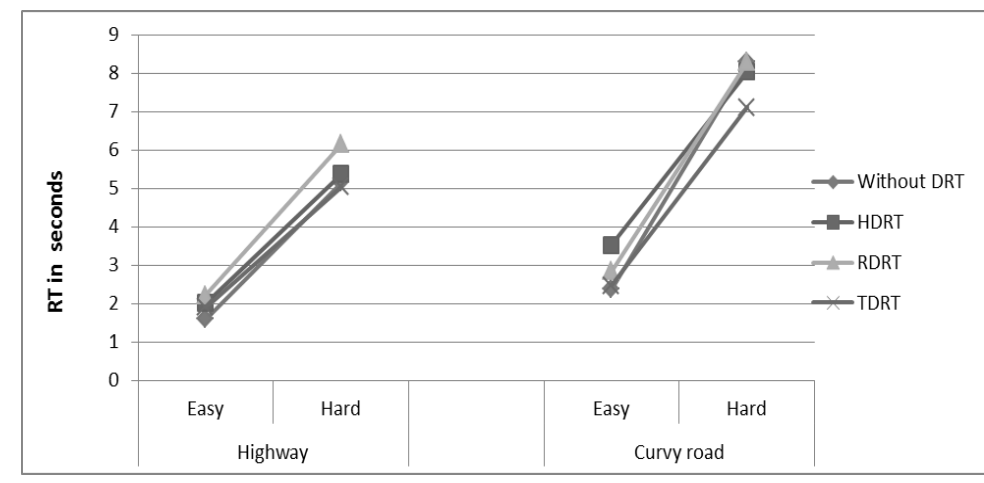

Figure 6. RT for each SuRT condition and each driving scenario

\section{Subjective evaluation of the difficulty to execute tasks simultaneously (Figure 7)}

Subjective evaluation of the ease to execute the tasks simultaneously was assessed by asking the participants to estimate the difficulty level on a 0-10 scale (from 0 no difficulty to 10 extremely difficult) after each drive.

First, a significant effect of the driving task demand was shown in terms of subjective evaluation of the difficulty to execute tasks simultaneously $[\mathrm{F}(1,15)=13.529, p=0.002)]$. Task management was found to be easier by the drivers in the highway than in the curvy road. A significant effect of DRT versions was also shown $[\mathrm{F}(3,13)=3.766, p=0.038)]$. The condition without any DRT was found easier than all DRT conditions $(p<0.05)$. No significant difference was found between DRT versions.

The tasks appeared to have different subjective levels of difficulty $[\mathrm{F}(4,12)=117.540$, $\mathrm{p}<0.001)]$. All of them differed significantly, and could be sorted as follow from the easiest to the most difficult task: Baseline, N-Back0, N-Back1, SuRT_easy, SuRT_hard ( $<<0.05)$. Moreover, a significant interaction between tasks and driving scenarios was highlighted showing that the increased difficulty for SuRT easy and hard was considered even greater in the curvy road scenario than in the highway scenario $[\mathrm{F}(4,12)=8.401, \mathrm{p}=0.002)]$. 


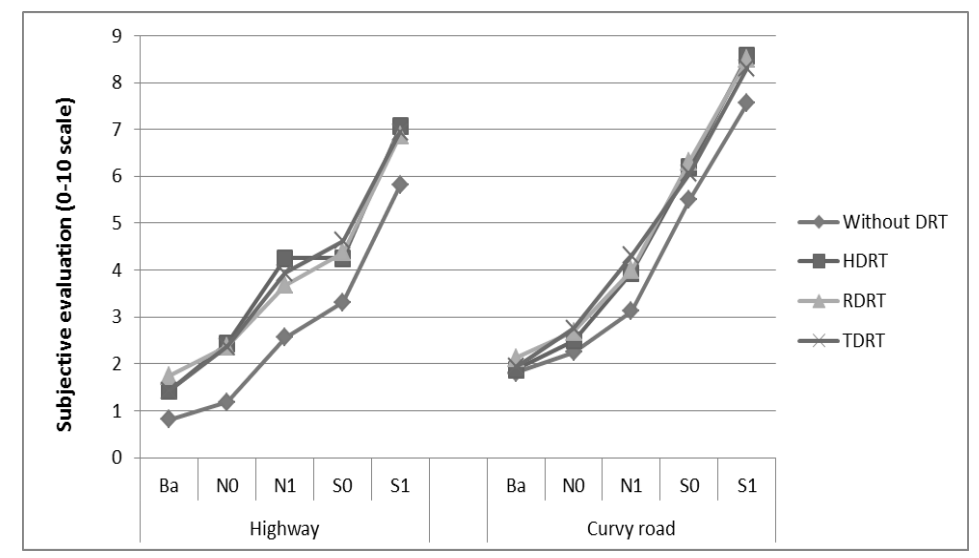

Figure 7. Subjective evaluation for each task and driving scenario

\section{DISCUSSION}

The results highlight a significant global effect of the driving demand on RTs to the DRT stimuli. When the driving demand is higher, in the curvy road scenario, RTs become larger. This result is confirmed by the drivers, which estimate that performing the three tasks simultaneously (driving while responding to secondary task and to DRT) is more difficult in the curvy road scenario than in the highway. Such a result is in line with other studies that have demonstrated DRT sensitivity to the driving demand using Peripheral Detection Task (Jahn and al., 2005; Martens and Van Winsum, 2000).

Concerning DRT Sensitivity to the task difficulty, results show that the more the triple task is considered as being difficult to perform, the more RTs to DRT stimuli increase. This is true for the N-Back tasks compared to the baseline, for N-Back1 compared to N-Back0, and also for the SuRT easy and hard when compared to the N-Back tasks and baseline conditions. However, it does not occur for SuRT hard as compared with SuRT easy, even if performing SuRT hard is considered as much more difficult than performing SuRT easy. These results clearly show that the DRT is sensitive to the levels of difficulty of a cognitive auditory task. However, they raise the question of its sensitivity to visual-manual tasks.

The subjective evaluation and SuRT performance provide elements to answer this question. The perceived difficulty increases between SuRT easy and SuRT hard reaching very high values for SuRT hard. That points out the great difficulties met by the drivers to accomplish the three tasks simultaneously and tend to indicate that they could reach the limits of their resources, given that cognitive load is extremely high in such situations. However, since that SuRT is a strategic and driver-paced task, it offers to them the possibility to compensate for this increased in workload by neglecting SuRT execution, in a way that preserve their performance on the DRT. This result raises the difficulty of measuring workload under multitasking conditions and the necessity to address how drivers prioritize attention towards the tasks. In this case, the participants received the instruction to give the priority to the driving task and to do their best to perform DRT and secondary tasks simultaneously. In the SuRT easy condition, they are more willing to execute the triple task and consequently their DRT performances are more affected than in the SuRT hard. In 
the later condition, they choose to allocate their attention in order to maintain their performance on the DRT, by allowing their SuRT performance to degrade.

However, such results also indicate a possible ceiling effect of the DRT sensitivity to discriminate between task levels of difficulty. As the DRT places drivers in a triple task situation, the method could reach its limit in evaluating tasks of too difficult levels, especially if they are self-paced such as the SuRT easy and hard. The next step will be to analyze the driving performances that will provide new elements to better understand the phenomenon.

\section{REFERENCES}

Engström, J., Aberg, N., Johansson, E \& Hammarbäck, J. (2005). Comparison between visual and tactile signal detection tasks applied to the safety assessment of in-vehicle information systems (IVIS). Proceedings of the Third International Driving Symposium on Human Factors in Driver Assessment, Training and Vehicle design, Rockport, Maine.

ISO TS14198. (2012). Road vehicles - Ergonomic aspects of transport information and control systems - Calibration tasks for methods which access driver demand due to the use of invehicle systems. ISO TC22/SC13/WG8.

ISO 16673. (2006). Road vehicles - Ergonomic aspects of transport information and control systems Occlusion method to assess visual distraction due to the use of in-vehicle systems. ISO TC22/SC13/WG8.

ISO 26022. (2010). Road vehicles- Ergonomics aspects of transport information and control systems simulated lane change test to asses in-vehicle secondary task demand. ISO TC22/SC13/WG8.

Jahn, G., Oehme, A., Krems, J. F., \& Gelau, C. (2005). Peripheral detection as a workload measure in driving: Effects of traffic complexity and route guidance system use in a driving study. Transportation Research Part F, 8, 255-275.

Martens, M.H., \& Van Winsum, W. (2000). Measuring distraction: the peripheral detection task, Soesterberg, Netherlands: TNO Human Factors.

Mattes, S., \& Hallén, A. (2009). Surrogate distraction measurement techniques: The Lane Change Test. In M. A. Regan, J. D. Lee \& K. L. Young (Eds.), Driver Distraction: Theory, Effects, and Mitigation. Boca Raton. FL: CRC Press Taylor \& Francis Group.

Mehler, B, Reimer, B \& Dusek, J.A. (2011). MIT AgeLab Delayed Digit Recall Task, Working paper 2011-3A.

Senders, J.W., Kristofferson, A.B., Levison, W.H., Dietrich, D.W., \& Ward, J.L. (1967). The Attentional Demand of Automobile Driving. Highway Research, 195, 15-33. 